\title{
The Stochastic Dynamics for Ecological Tourism System with Visitor Educational Intervention
}

\author{
Dongping Wei, ${ }^{1,2}$ Shouwen Wen, ${ }^{3}$ Yizeng Chen, ${ }^{1}$ Yong Xu, ${ }^{4}$ and Bing Liang ${ }^{2}$ \\ ${ }^{1}$ School of Management, Shanghai University, Shanghai 200444, Shanghai, China \\ ${ }^{2}$ Math and Physics Department, Shenzhen Polytechnic, Shenzhen 518055, Guangdong, China \\ ${ }^{3}$ School of Management, Shenzhen Polytechnic, Shenzhen 518055, Guangdong, China \\ ${ }^{4}$ School of Mathematical Sciences and Computing Technology, Central South University, Changsha 410075, Hunan, China \\ Correspondence should be addressed to Yizeng Chen; shucyz@163.com
}

Received 28 March 2013; Accepted 16 April 2013

Academic Editor: J. A. Tenreiro Machado

Copyright (c) 2013 Dongping Wei et al. This is an open access article distributed under the Creative Commons Attribution License, which permits unrestricted use, distribution, and reproduction in any medium, provided the original work is properly cited.

\begin{abstract}
The ever-increasing visitation in parks and protected areas continues to present a considerable challenge for worldwide land managers with allowing recreational use while preserving natural conditions. In China, the fast expanding visitation in protected areas is quickly damaging the natural resources and precious culture without effective visitor education, while regulation and site management are also gaining very limited efficacy. We propose a differential equation to describe the ecological tourism system. Shown by the theoretical proof and numerical simulation, the ecological tourism system is unstable without any perturbed factors, especially visitor educational intervention, because the solution of the dynamic system explodes in a finite time given any initial value. Supposing that the intrinsic increasing rate of stakeholders in the systems stochastically perturbed by the visitor educational intervention, we discover that the stochastic dynamic model can effectively suppress the explosion of the solution. As such, we demonstrate that the tourism system can develop steadily and safely even under a large amount of visitors in public vacation, when employing continuous visitor education intervention programmes.
\end{abstract}

\section{Introduction}

Currently, the Chinese tourism market is the primary domestic tourism market owning about 1.7 billion visitors per year and the fourth inbound tourism market and the primary outbound tourism market in the Asian-Pacific region owning about 45.84 million visitors per year [1]. Many scholars proclaim in surprise that the era of recreation is approaching ordinary Chinese daily life. In the public holidays such as golden week of Labor's day and National day, it is crowded and chaotic in every popular scene site such as Huangshan Mountain, Huashan Mountain, and the Great Wall, In these crowded parks or natural protected areas, the negative impacts, such as litter, tree damage, noise, and the rude behaviors of visitors, shock the environmentalists. In the United States, scholars concluded that the undesirable impacts on the tourism destination with fast expanding visitation include resource degradation and social impacts $[2,3]$. The resource impacts associated with large amount of visitors include the trampled vegetation and soil damage, soil compaction and erosion, litter, human waste, and wildlife disturbance. The social impacts include the diminished satisfaction of visitors and recreation conflicts between visitors, visitors and park managers, visitors and neighbors of tourism destinations, and the culture conflicts [4]. Ferreira and Rosso [5] discovered that the Sao Paulo coast is highly depredated by the increased tourist activity. Manning and Aderson [6] also found that the dramatically grown visitors' use of Arches National Park has had several important impacts on the park including trampling of fragile soils and vegetation (impacts on soil and vegetation) and crowding on trails and at attraction sites (crowding, impacts on trails and attraction sites). Left unmanaged, these impacts can lead to unacceptable changes in resource and social conditions such as the loss of sensitive or rare plants and animals, or declines in visitor satisfaction [7]. In China, many visitors feel awful when they arrive the tourism destination because of crowded people and the rude behaviors. 
Facing the increasingly serious negative tourism impacts, many scholars propose different strategies to reduce the impacts. Ferreira and Rosso [5] proposed that the management strategies should include isolation of sensitive areas, construction of boardwalks, visitor education, and monitoring programmes to reduce the degradation of rocky shore fauna on the Sao Paulo coast caused by the development of tourism. In United States, many park managers commonly employ regulations, site management, and visitor education to address the resource and social impacts. The regulations directly curtail tourism freedom and antagonize visitors by their enforcement. But the enforcements of regulations are often costly and lack efficacy. Peterson and Lime [8], Hendee and Dawson [3], and Stankey and Schreyer [9] noted that site management actions were less direct but they were also costly and permanently altered the natural setting and the nature of recreational experiences. The visitor education is a more appropriate, light-handed and indirect management response to reduce resource and social impacts $[4,10]$. Docutte and Cole [11] presented that visitor education has been widely touted as the most appropriate approach to managing recreation in wilderness. Visitor education is also easier to win the support of visitors [3]. Hence, in the United States, the federal land management agencies employed a Leave No Trace (LNT) programme for visitor education in order to reduce the resource and social impacts. And many other international organizations such as Members of the World Tourism Organization developed a Global Code of Ethics for Tourism in 1999 to help minimize the negative impact of tourism on the environment and the cultural heritage (WTO, 2006). The Ecotourism society (1993) has also put forward "Ecotourism Guidelines for Nature Operators" to guide the visitor educational efforts to minimize the social and environment impacts. Hendee and Dawson [3] concluded that effective educational programmes can (1) reduce per capita and cumulative resource and experiential impacts; (2) aid in keeping resource and social conditions within acceptable limits; (3) reduce the need for site management on regulatory actions such as limits; (4) provide a key component of sustainable tourism programmes.

There are a lot of references to study the visitor educational message content, message delivery approaches, audience characteristics and theoretical grounding including moral development theory and decisionmaking theory, reasoned actions and planned behavior theory. However, there is little research to study the relationship between the stakeholders and the human ecological system of tourist destinations. In fact, the stakeholders in ecotourism destinations cannot survive without the support from one another, and they must unite to a population in a certain space [12]. In 2012, Shou-wen started to analyze the dynamic property of visitors and the ecological tourism system under the visitor educational intervention. Shou-wen et al. [13] set up the stochastic increasing model of stakeholders perturbed by the continuous visitor education. In this paper, we make efforts to the further study to better understand the relationship between the stakeholders and ecological tourism system intervened by visitor educational programmes. Based on stochastic differential equation theory [14], we explore whether the ecological tourism system can develop sustainably when perturbed by the continual visitor educational intervention.

In order to clearly present the mathematical models in the next three sections, we introduce the key definitions and notations of variables in the following.

$x(t)$ : stakeholders in an ecological tourism system at time $t$.

$x_{0}$ : the initial value of differential equation at time $t_{0}$.

$\alpha$ : the intrinsic increasing rate of stakeholders within the ecological tourism system.

$\beta$ : the immigration rate of stakeholders from outside the system.

$\gamma$ : the emigration rate of stakeholders from the inner system.

$K$ : the maximum carrying capacity of the ecological tourism system.

$\omega(t)$ : the Brownian movement.

$\varepsilon$ : the intensity of visitor education intervention.

$\tau_{e}$ : the explosion time of stochastic differential equation.

$\tau_{k}$ : the stopping time of stochastic differential equation.

$k_{0}$ : a sufficiently large value.

$k$ : any nonnegative integer.

$V(x)$ : a $C^{2}$-function, $V: R^{+} \rightarrow R^{+}$.

$M$ : a sufficient large positive value.

$T$ : a sufficient large positive constant.

\section{The Logical Differential Equation Model of Ecological Tourism System}

In the literature, the ecological tourism system is defined as all the natural resources including air, rocks, mountains, soils, trees, wildlife, and all the stakeholders in visitation destination. And the stakeholders [12] in ecotourism include the seven factors: (1) the tourism resource suppliers' group such as tourism resource managers or park managers; (2) the visitation product sellers group including tourism company, and tourism website managers; (3) the group of relative products or services suppliers who are providing transportation and hotels, catering, other tourism memories products; (4) the group of local communities including the staff of tourism companies and workers in other company and local people; (5) the group of governments including super government organizations and centeral and local governments; (6) the special interest group including nongovernment organizations like environmental organizations, human rights and labor rights organizations, or trust and environmental charity institutes, academics, and social medias; and (7) the core tourism products and services consumers like visitors.

We supposed that there are $x(t)$ stakeholders in an ecological tourism system at time $t$, considering that there are some stakeholders entering the system from outside the ecological 
system and some others leave the system when they finish their activities or business. We denote $\alpha$ as the intrinsic increasing rate of stakeholders within the ecological tourism system, $\beta$ as the immigration rate of stakeholders from outside the system, and $\gamma$ as the emigration rate of stakeholders from the inner system. $K$ stands for the maximum carrying capacity of the ecological tourism system. And then $1 / K$ represents the recreation impact factors per capita. So $x(t) / K$ means the recreation impact factor at time $t$. So mapping the logistical differential equation on the ecological system, we have

$$
\frac{d x}{d t}=x\left(\alpha+\beta-\gamma-\frac{1}{K} x\right)
$$

It is the original differential equation to describe the relationship between the stakeholders and the ecological system.

Theorem 1. For any given initial value $x_{0}$, the solution of (1) $x(t)$ cannot avoid an explosion in a finite time.

Proof. Since the variable $x(t)$ denotes the population of stakeholder, $x(t)$ must be a positive value at time $t \geq 0$. When the parameters of (1) $\alpha, \beta, \gamma \in(0,1), K \in R^{+}$, (1) only has a local solution as follows

$$
x(t)=\frac{\alpha+\beta-\gamma}{-(1 / K)+e^{-(\alpha+\beta-\gamma) t}\left(\alpha+\beta-\gamma-(1 / K) x_{0}\right) / x_{0}} .
$$

Given that $x_{0}>0$, it is not difficult to conclude that $x(t)$ explodes to infinity when $t \rightarrow \mathscr{T}$,

$$
\mathscr{T}=-\frac{1}{\alpha+\beta-\gamma} \log \frac{-(1 / K) x_{0}}{\left(\alpha+\beta-\gamma-(1 / K) x_{0}\right)} .
$$

Hence, the solution of (1) $x(t)$ will explode to infinity in a finite time.

In other words, the original differential equation model just has the local solution. The simulation data in Table 1 and Figure 1 show that the dynamic model (1) explodes in a finite time for any initial value $x_{0}=30,40,50,70,80,100$, when $\alpha=0.25, \beta=0.12, \gamma=0.08$. and $K=1000$. For instance, according to simulation data in Table 1 and Figure 1, we can easily find that the number of stakeholders $x(10)=1081$ is a little larger than the maximum capacity of the system $K=1000$, but at time $t=11, x(11)=2990$ is much larger than $K=1000$, when the initial value of stakeholder $x_{0}=$ 40 without any control on the system. Hence, the system is chaotic and unsustainable after $t=10$. Eventually, the system is collapsed at time $t=12$, because $x(12)=-5679<0$. When $x_{0}=30,40,50,70,80,100$, the system is collapsed at time $t=14,12,10,7,6,4$, see Figure 1; therefore, it can be concluded that the system is collapsed faster with larger initial value $x_{0}$.

Similarly, Figure 2 demonstrates that the explosion of the dynamic model (1) cannot avoid any initial value $x_{0}=30,40$, $45,60,70,80$, given that $\alpha=0.15, \beta=0.08, \gamma=0.06$, and $K=1000$. For instance, Table 2 shows that the number of stakeholders $x(16)=1698$ is much larger than the maximum
TABLE 1: The simulation of $x(t)$ given the different initial value $x_{0}$.

$$
x(t)
$$

\begin{tabular}{lcccccc}
$t$ & \multicolumn{5}{c}{$\alpha=0.25, \beta=0.12, \gamma=0.08, K=1000$} \\
\hline 1 & $x_{0}=30$ & $x_{0}=40$ & $x_{0}=50$ & $x_{0}=70$ & $x_{0}=80$ & $x_{0}=100$ \\
2 & 53 & 79 & 112 & 215 & 301 & 688 \\
3 & 63 & 95 & 138 & 280 & 415 & 1262 \\
4 & 75 & 116 & 172 & 382 & 617 & 4545 \\
5 & 91 & 143 & 219 & 555 & 1069 & -3635 \\
6 & 110 & 179 & 287 & 916 & 2907 & -1422 \\
7 & 135 & 229 & 392 & 2087 & -5956 & -931 \\
8 & 169 & 302 & 575 & -19651 & -1638 & -717 \\
9 & 215 & 417 & 961 & -1963 & -1006 & -598 \\
10 & 281 & 622 & 2303 & -1104 & -755 & -523 \\
11 & 382 & 1081 & -11137 & -800 & -621 & -472 \\
12 & 556 & 2990 & -1841 & -647 & -538 & -435 \\
13 & 918 & -5679 & -1069 & -555 & -482 & -408 \\
14 & -18836 & -1000 & -638 & -451 & -413 & -370 \\
15 & -1956 & -752 & -549 & -420 & -391 & -357 \\
16 & -1102 & -619 & -490 & -396 & -373 & -346 \\
17 & -800 & -537 & -448 & -377 & -359 & -337 \\
18 & -646 & -481 & -417 & -362 & -348 & -330 \\
19 & -554 & -442 & -394 & -351 & -339 & -324 \\
\hline
\end{tabular}

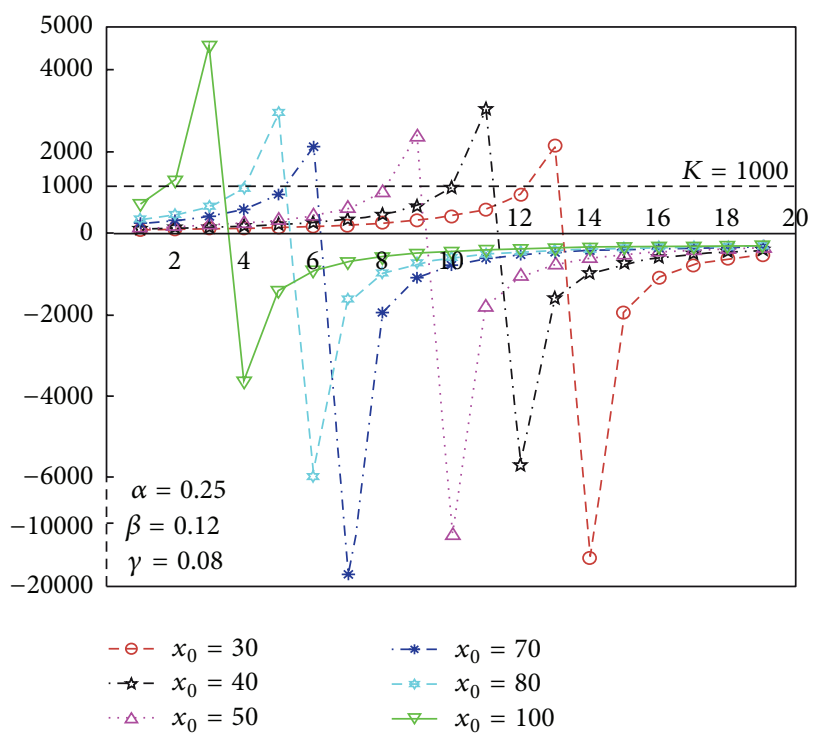

FIgURE 1: The simulation of $x(t)$ given the different initial values of $x_{0}$.

capacity of $K=1000$, given the initial value of stakeholder $x_{0}=30$ without any control on the system. Hence, the system is chaotic and unsustainable after time $t=16$. Eventually, the system will be collapsed at time $t=18$. When $x_{0}=30$, $40,45,60,70,80$, the system is collapsed at time $t=18,13$, $12,10,6,3$, see Figure 1 . Therefore, it can be concluded that the system is collapsed faster with larger initial value $x_{0}$. 
TABLE 2: The simulation of $x(t)$ given the different initial value $x_{0}$ and $\alpha, \beta$, and $\gamma$.

\begin{tabular}{lcccccc}
\hline \multicolumn{6}{c}{$x(t)$} \\
$t$ & \multicolumn{5}{c}{$\alpha=0.15, \beta=0.08, \gamma=0.06, K=1000$} \\
\hline 1 & $x_{0}=30$ & $x_{0}=40$ & $x_{0}=45$ & $x_{0}=60$ & $x_{0}=70$ & $x_{0}=80$ \\
2 & 58 & 98 & 127 & 166 & 311 & 828 \\
3 & 65 & 112 & 148 & 198 & 404 & 1588 \\
4 & 73 & 129 & 174 & 240 & 558 & 10110 \\
5 & 83 & 151 & 208 & 299 & 856 & -2574 \\
6 & 94 & 179 & 254 & 385 & 1685 & -1196 \\
7 & 108 & 214 & 319 & 525 & 15181 & -802 \\
8 & 125 & 263 & 417 & 787 & -2388 & -615 \\
9 & 145 & 332 & 581 & 1456 & -1157 & -507 \\
10 & 171 & 437 & 908 & 6590 & -786 & -437 \\
11 & 204 & 616 & 1878 & -2942 & -607 & -387 \\
12 & 249 & 989 & 102870 & -1263 & -502 & -351 \\
13 & 312 & 2224 & -2126 & -829 & -433 & -323 \\
14 & 405 & -15070 & -1097 & -630 & -384 & -301 \\
15 & 559 & -1851 & -760 & -516 & -349 & -283 \\
16 & 1690 & -1025 & -592 & -443 & -321 & -268 \\
17 & 16191 & -574 & -427 & -354 & -282 & -246 \\
18 & -2366 & -481 & -380 & -325 & -268 & -237 \\
19 & -1153 & -418 & -345 & -303 & -256 & -230 \\
\hline & & & & & &
\end{tabular}

Comparing with Figure 1, Figure 2 indicates that the system is collapsed slower with smaller parameters $\alpha, \beta$, and $\gamma$.

The visitors $x_{0}$ are very large in some protected areas especially on the public vacations in China. So the solution of the differential dynamic equation will inevitably explode without any interventional programme. The tourism system of some famous protected areas or precious fragile natural history park is currently in the great danger of large visitations.

\section{The Stochastically Dynamics Model Perturbed by Visitor Educational Intervention}

As demonstrated in the simulations of Section 2, with the high and disordered consumption, the ecological tourism system especially precious fragile protected areas or parks will eventually collapse without any visitor education or other visitor interventions. The booming China tourism market is extremely crowded in many famous tourism destinations on the public vacations. Although there are a lot of visitor regulations and rules in the tourism destinations, the lack of the effective visitors educational intervention coused the natural resource and culture resource to be badly degraded. The natural resource impacts and social impacts are extremely serious. So it is critical importance to develop effective and systematical visitor educational programme such as the Chinese version of LNT Programme to avoid systematical

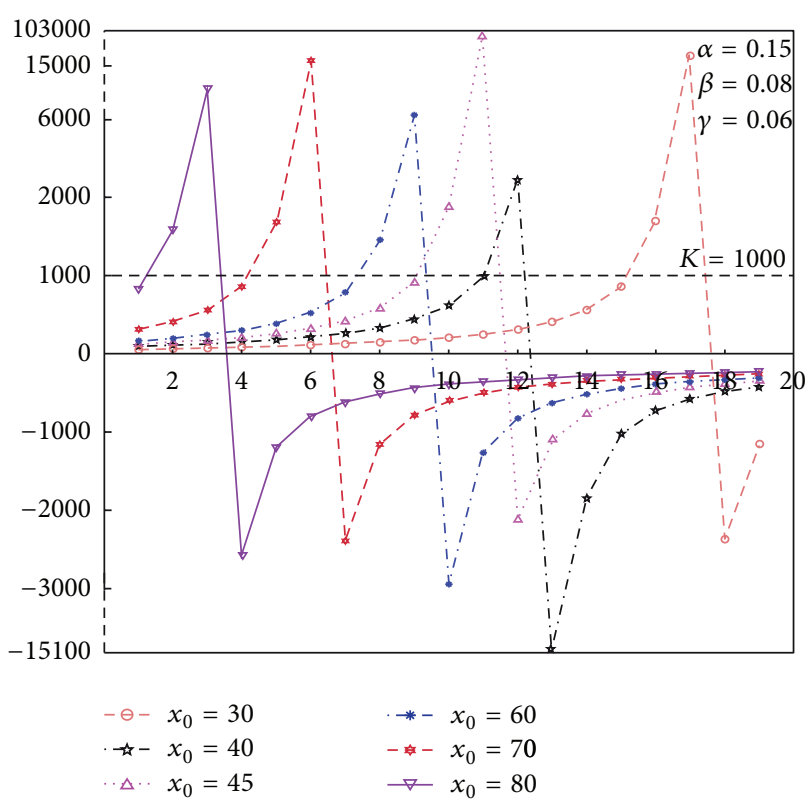

FIGURE 2: The simulation of $x(t)$ given the different initial values of $x_{0}$ and $\alpha, \beta$, and $\gamma$.

explosion of the ecological tourism system. Increasing tourist activity in protected regions demands management strategies including visitor education to reduce the impacts on the ecological tourism system and avoid the explosion of solution of the dynamic equation describing the system. In this paper, we suppose that the intrinsic increasing rate of stakeholders is intervened by visitor education, but the efficacy of visitor education is different from diverse visitor characteristics, the educational message content, message delivery approaches, and so forth. Hence, the intrinsic increasing rate of stakeholders behaves stochastically when perturbed by visitor intervention. Naturally, we can suppose that the intrinsic rate of increase is stochastically perturbed with

$$
\alpha \longrightarrow \alpha+\varepsilon \omega(t),
$$

where $\omega(t)$ and $\varepsilon$ represent the Brownian movement and the intensity of visitor education, respectively. Substituting (4) into the original differential equation (1), we can obtain the stochastic dynamic model of ecological tourism system perturbed by visitor education intervention programme,

$$
d x=x\left(\alpha+\beta-\gamma-\frac{1}{K} x\right) d t+\varepsilon x^{2} d \omega(t) .
$$

The critical purpose of the paper is to study whether the stochastic parameter of intrinsic increasing rate of stakeholders can effectively suppress the explosion of the solution of (2) in a finite time with any initial value $x_{0}$ and stop the collapse of the ecological tourism system eventually. In this paper, we should prove that the solution of (5) can no longer explode in a finite time with a probability one when $\alpha+\beta<\gamma+(1 / K) x_{0}$. And we will then see that for arbitrary parameters $\alpha, \beta, \gamma \in$ $[0,1]$ and $K \in R^{+}$, the dynamic system will not explode in a finite time with a probability one provided any intensity of 
the perturbed visitor educational intervention $\varepsilon$. The results demonstrate that effective visitor education programmes will support the sustainable development of ecological tourism system and suppress the explosion of the stochastic dynamic model.

\section{The Existence of Nonexplosion Solutions of the Ecological Tourism Dynamic System with Visitor Education of Intervention}

In the paper, we denote $\left(\Omega, \mathscr{F},\left\{\mathscr{F}_{t}\right\}_{t \geq 0}, \mathscr{P}\right)$ to be a complete probability space with a filtration $\left\{\mathscr{F}_{t}\right\}_{t \geq 0}$ satisfying the usual condition (i.e., right continuous, and $\mathscr{F}_{0}$ contains all $p$-null sets) [14]. Let $\omega(t)$ be one-dimensional Brownian motion defined on the probability space.

The definition $x(t)$ should be nonnegative because it represents the stakeholders. For any given initial value $x_{0}$, if the stochastic differential equation (5) has a unique global solution, then the coefficient of the equation should be satisfy the linear growth condition and local Lipschilz condition [14-17]. Unfortunately, the coefficients of (5) only satisfy the locally Lipschitz continuous condition, so the solution of (5) only has local solution which may explode at a finite time [18].

Now, we will show that the visitor education intervention can suppress the explosion of the solution as proved by the following theorem.

Theorem 2. Given any system parameters $\alpha, \beta, \gamma \in[0,1], K \in$ $R^{+}$and any initial value $x_{0} \in R^{+}$if intensity of visitor education intervention $\varepsilon \neq 0$, there is a unique solution $x(t)$ of (3) at $t \geq 0$, and the solution will be positive with probability one, namely, $x(t)>0$ when $t \geq 0$ a.s.

Proof. For the coefficients of (5) are the locally Lipschitz continuous, for any given initial value $x_{0} \in R^{+}$, there is a unique solution $x(t)$ on the $t \in\left(0, \tau_{e}\right)$ where $\tau_{e}$ is the explosion time $[18,19]$. To prove the solution of $(5) x(t)$ is global and positive, we need to show that $\tau_{e}=\infty$ almost surely. Let $k_{0}>0$ be sufficiently large value and the initial value of the equation $x_{0}$ belong to the interval $\left[1 / k_{0}, k_{0}\right]$. For any nonnegative integer $k \geq k_{0}$, we define the stopping time as follows

$$
\tau_{k}=\inf \left\{t \in\left[0, \tau_{e}\right): x(t) \notin\left(\frac{1}{k}, k\right)\right\},
$$

where setting $\inf \emptyset=\infty$ throughout the whole paper, as usual $\emptyset$ denotes the empty set. Obviously, $\tau_{k}$ is increasing when $k \rightarrow \infty$. Set $\tau_{\infty}=\lim _{k \rightarrow \infty} \tau_{k}$ hence $\tau_{\infty} \leq \tau_{e}$ a.s. If it can be proved that $\tau_{\infty}=\infty$ a.s., then it is natural that $\tau_{e}=\infty$ a.s. and $x(t) \in R^{+}$for any $t \geq 0$. In other words, in order to show that $x(t) \in R^{+}$will not explode, we should show that $\tau_{\infty}=\infty$ a.s. first. Employing contradiction methodology, if this statement $\tau_{\infty}=\infty$ a.s. is false, then there is a pair of constant $T \geq 0$ and $\varepsilon \in(0,1)$, such that

$$
P\left\{\tau_{\infty} \leq T\right\}>\varepsilon .
$$

Therefore, an integer $k_{1} \geq k_{0}$ can be found, such that

$$
P\left\{\tau_{\infty} \leq T\right\}>\varepsilon \quad \forall k \geq k_{1} .
$$

Define a $C^{2}$-function $V: R^{+} \rightarrow R^{+}$,

$$
V(x)=\sqrt{x}-1-\log x^{0.5} .
$$

It is easily proved that $C^{2}$-function $V(x)$ is nonnegative on $x>0$. follows

If $x(t) \in R^{+}$, it can be calculated from the Ito formula as $d V(x)$

$$
\begin{aligned}
= & \left\{0.5\left(x^{-0.5}-x^{-1}\right) x\left[\left(\alpha+\beta-\gamma-\frac{1}{K} x\right) d t+\varepsilon x d \omega(t)\right]\right\} \\
& +0.5\left(0.5 x^{-2}-0.25 x^{-1.5}\right) \varepsilon^{2} x^{4} d t \\
= & \left\{0 . 5 ( x ^ { 0 . 5 } - 1 ) \left[\left(\alpha+\beta-\gamma-\frac{1}{K} x\right)\right.\right. \\
& \left.\left.+\left(0.25-0.125 x^{0.5}\right) \varepsilon^{2} x^{2}\right]\right\} d t \\
& +0.5\left(x^{0.5}-1\right) \varepsilon x d \omega(t),
\end{aligned}
$$

where we denote $x(t)=x$ for simplification.

Then (10) can be simplified as follows

$$
\begin{aligned}
& 0.5\left(x^{0.5}-1\right)\left(\alpha+\beta-\gamma-\frac{1}{K} x\right) \\
&= 0.5(\alpha+\beta-\gamma) x^{0.5}-0.5 \frac{1}{K} x^{1.5} \\
&-0.5(\alpha+\beta-\gamma)+0.5 \frac{1}{K} x \\
&=-0.5 \frac{1}{K} x^{1.5}+0.5 \frac{1}{K} x \\
&+0.5(\alpha+\beta-\gamma) x^{0.5}-0.5(\alpha+\beta-\gamma), \\
&(0.25-\left.0.125 x^{0.5}\right) \varepsilon^{2} x^{2}=0.25 \varepsilon^{2} x^{2}-0.125 \varepsilon^{2} x^{2.5} .
\end{aligned}
$$

We note that

$$
\begin{aligned}
F(x) & \\
= & -0.125 \varepsilon^{2} x^{2.5}+\frac{1}{4} \varepsilon^{2} x^{2}-0.5 \frac{1}{K} x^{1.5} \\
& +0.5 \frac{1}{K} x+0.5(\alpha+\beta-\gamma) x^{0.5}-0.5(\alpha+\beta-\gamma) \\
\leq & -0.125 \varepsilon^{2} x^{2.5}+\frac{1}{4} \varepsilon^{2} x^{2}+0.5 \frac{1}{K} x^{1.5} \\
& +0.5 \frac{1}{K} x+0.5(\alpha+\beta-\gamma) x^{0.5}+0.5(\alpha+\beta-\gamma) .
\end{aligned}
$$

Then we have $d V(x)=F(x) d t+0.5\left(x^{0.5}-1\right) \varepsilon x d \omega(t)$.

If $\varepsilon \neq 0$, then it is not difficult to yield that $F(x)$ is bounded by sufficiently large positive value $M$, so that $d V(x) \leq M+$ $0.5\left(x^{1.5}-x\right) \varepsilon d \omega^{T}(t)$. 
It is clear to obtain the following expression by integrating the both sides of the proceeding inequality from 0 to $T \wedge \tau_{k}$ as follows

$$
\int_{0}^{T \wedge \tau_{k}} d V(x) \leq \int_{0}^{T \wedge \tau_{k}} M d t+\int_{0}^{T \wedge \tau_{k}} 0.5\left(x^{0.5}-1\right) \varepsilon x d \omega(t) .
$$

With $\left(T \wedge \tau_{k}\right) \in R^{+}$, taking expectations on both sides of inequality (8), we can yield

$$
E V\left(x\left(T \wedge \tau_{k}\right)\right) \leq V\left(x_{0}\right)+M E\left(T \wedge \tau_{k}\right) \leq V\left(x_{0}\right)+M T .
$$

Let $\Omega_{k}=\left\{\tau_{k} \leq T\right\}$ for $k \geq k$ and the inequality (7) so that $P\left(\Omega_{\kappa}\right)>\varepsilon$. Taking any $w \in \Omega_{\kappa}$, with the definition of stopping time $(6), x\left(\tau_{k}, w\right)$ equals either $1 / k$ or $k$, and then it is easy to conclude that $V\left(x\left(\tau_{k}, w\right)\right)$ is not less than either $\sqrt{k}-1-0.5 \log k$ or $\sqrt{1 / k}-1-0.5 \log (1 / k)$. So $x(t) \geq k$ or $x(t) \leq 1 / k$ when $t \geq \tau_{k}$. Therefore, it is not difficult to get the following inequality:

$$
\begin{aligned}
& V\left(x\left(\tau_{k}, w\right)\right) \\
& \quad \geq[\sqrt{k}-1-0.5 \log k] \wedge \sqrt{\frac{1}{k}}-1-0.5 \log \left(\frac{1}{k}\right) .
\end{aligned}
$$

Taking expectation on both sides of (15), we get

$$
\begin{aligned}
& E V\left(x\left(\tau_{k}, w\right)\right) \\
& \quad \geq[\sqrt{k}-1-0.5 \log k] \wedge \sqrt{\frac{1}{k}}-1-0.5 \log \left(\frac{1}{k}\right) .
\end{aligned}
$$

It is easy to show that

$$
\begin{aligned}
V\left(x_{0}\right) & +M T \\
& \geq E\left[1_{\Omega_{\hbar}(w)} V\left(x\left(T \wedge \tau_{k}\right)\right)\right] \\
& \geq \varepsilon[\sqrt{k}-1-0.5 \log k] \wedge \sqrt{\frac{1}{k}}-1-0.5 \log \left(\frac{1}{k}\right),
\end{aligned}
$$

where $1_{\Omega_{k}(w)}$ is the indicator function of $\Omega_{\kappa}$. Letting $k \rightarrow \infty$ in (17), then

$$
\infty>V\left(x_{0}\right)+M T=\infty,
$$

where $T$ is a constant denoted in (7). Obviously, (18) is contradicted. So we can conclude that the statement of (7) is false. Hence, there are constants $T>0$ and $\varepsilon \in(0,1)$ such that $P\left\{\tau_{\infty} \leq T\right\}>\varepsilon$. Consequently, the original statement is true in which $P\left\{\tau_{k} \leq \tau_{\infty}\right\}<\varepsilon$. In other words, the statement $\tau_{\infty}=\infty$ is true almost surely.

It is clear to derive from the Theorem 2 that there is a positive solution $x(t)$ of $(7)$ and $x(t)$ will never explode in a finite time. Following the condition of Theorem 2, we can deduce that the visitor educational intervention brings the stochastic intrinsic increasing rate of stakeholders into the tourism ecological system. When the intensity of intervention $\varepsilon \neq 0$, the stochastic intrinsic increasing rate can effectively suppress the explosion of the solution $x(t)$ for any given $x_{0}$ and avoid the collapse mentioned in Section 2. It means that the slight or tiny stochastical change of intrinsic increasing rate of stakeholder introduced by visitor educational intervention can keep the tourism ecological system developing safely and steadily. In fact, early in 1970s, Fishbein, Fazio [20], and Bradley [21] already noticed that the negative recreation impacts on wildlands and wildlife, and the ecological system or environment could not keep sustainable development without recreation planning and management. According to Theorem 2, the visitor educational intervention is a necessary condition for the safe and stable developing of the tourism ecological system. Especially in China, the booming recreation market is in urgent need of the visitor educational intervention to stop the chaotic tourism activities.

\section{The Boundedness of the Solution of the Dynamic Model}

With the intensities of the visitor educational intervention, the solution of the dynamic model (5) cannot explode in a finite time following by the Theorem 2 . However, it is not enough to show that the solution exists and does not explode. We consider how the solution of the dynamic model varies in a finite interval in this section. In other words, we will show that the solution of (5) is ultimately bounded.

Denote $x\left(t, x_{0}\right)$ as the unique global solution of (5) for any given initial value $x_{0}>0$. We define stochastically ultimate bounded property of the solution of the dynamic model as follows.

Definition 3. For any initial value $x_{0}>0$, if the solution of (3) $x\left(t, x_{0}\right)$ has the property

$$
\lim _{t \rightarrow \infty} \inf x\left(t, x_{0}\right)>0
$$

then (5) is defined to be stochastically ultimately bounded with probability one. The following theorem shows (5) is stochastically ultimately bounded.

Theorem 4. If the coefficient of (5) satisfies the following inequality:

$$
\begin{gathered}
\frac{1}{2} \varepsilon^{2}-\frac{1}{K}<0 \\
\left(\alpha+\beta-\gamma+\frac{1}{K}\right)^{2}+4\left(\frac{1}{2} \varepsilon^{2}-\frac{1}{K}\right)(\alpha+\beta-\gamma) \leq 0 .
\end{gathered}
$$

Then the solution of (5) $x\left(t, x_{0}\right)$ is stochastically ultimately bounded with a probability one. Given any initial value $x_{0} \in$ $R^{+}$, the solution of (3) has the property

$$
\lim _{t \rightarrow \infty} \sup x\left(t, x_{0}\right)<\infty
$$


So the solution of $(5) x\left(t, x_{0}\right)$ belongs to a finite interval

$$
\left[\lim _{t \rightarrow \infty} \inf x(t), \lim _{t \rightarrow \infty} \sup x(t)\right] .
$$

Proof. It is necessary to define a $C^{2}$-function $V: R^{+} \rightarrow R^{+}$ by

$$
V(x)=x-1-\log x .
$$

Applying the Ito formula, we derive from (23) that

$$
d V(x)=L V(x) d t+\left(1-x^{-1}\right) x^{2} \varepsilon d \omega(t),
$$

where

$$
\begin{aligned}
L V(x) & =\left(1-x^{-1}\right) x\left(\alpha+\beta-\gamma-\frac{1}{K} x\right)+\frac{1}{2} x^{-2} \varepsilon^{2} x^{4} \\
& =(x-1)\left(\alpha+\beta-\gamma-\frac{1}{K} x\right)+\frac{1}{2} \varepsilon^{2} x^{2} \\
& =\left(\frac{1}{2} \varepsilon^{2}-\frac{1}{K}\right) x^{2}+\left(\alpha+\beta-\gamma+\frac{1}{K}\right) x-(\alpha+\beta-\gamma) .
\end{aligned}
$$

It is obvious to see that $L V(x(t))$ is a quadratic function. Applying the property of quadratic function, when

$$
\begin{gathered}
\frac{1}{2} \varepsilon^{2}-\frac{1}{K}<0 \\
\Delta=\left(\alpha+\beta-\gamma+\frac{1}{K}\right)^{2}+4\left(\frac{1}{2} \varepsilon^{2}-\frac{1}{K}\right)(\alpha+\beta-\gamma) \leq 0 .
\end{gathered}
$$

If (20) is satisfied, the quadratic function $L V(x(t))$ must be less than zero. Therefore, we can get that

$$
d V(x(t)) \leq 0 d t+\left(x^{2}-x\right) \varepsilon d \omega(t) .
$$

Integrating both sides of inequality (28) from 0 to $t$, we can get

$$
\int_{0}^{t} d V(x(t)) \leq \int_{0}^{t} 0 d t+\int_{0}^{t}\left(x^{2}-x\right) \varepsilon d \omega(t) .
$$

Thus,

$$
V(x(t)) \leq V\left(x_{0}\right)+\int_{0}^{t}\left(x^{2}-x\right) \varepsilon d \omega(t) .
$$

For convenience, we define

$$
X(t):=V\left(x_{0}\right)+\int_{0}^{t}\left(x^{2}-x\right) \varepsilon d \omega(t) .
$$

Since $V(x)$ is a nonnegative function, applying Doob's martingale convergence theorem, we can yield

$$
\lim _{t \rightarrow \infty} x(t)<\infty \quad \text { a.s. }
$$

Thus, it is natural to yield $\lim _{t \rightarrow \infty} \sup x(t)<\infty$ a.s.

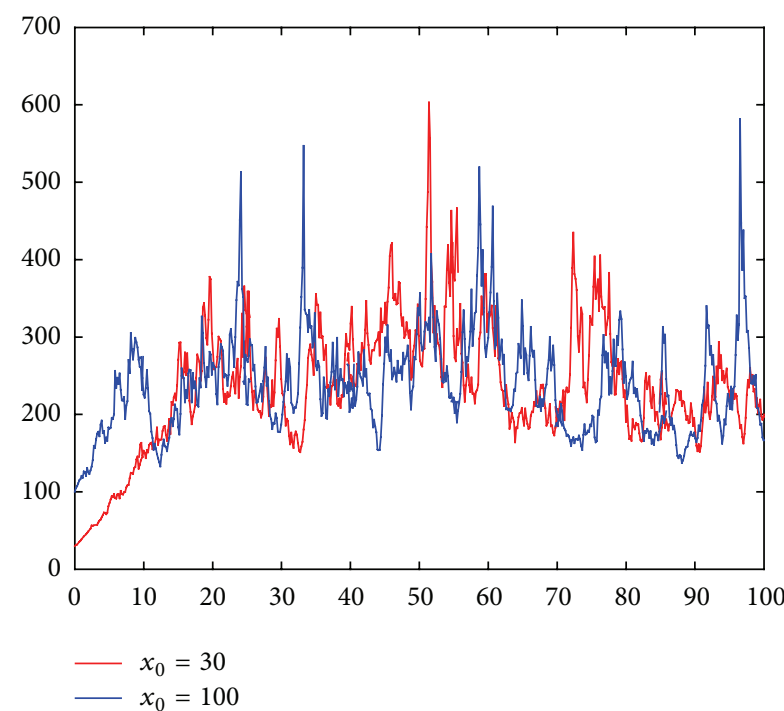

FigURE 3: The simulation of $x(t)$ with $\alpha=0.25, \beta=0.12, \gamma=0.08$, and $K=1000$ and different initial values $x_{0}$.

Recalling the definition of $C^{2}$-function $V: R^{+} \rightarrow R^{+}$, if and only if $x \rightarrow \infty$ or $x \rightarrow 0$, the limit of $V(x)=x-1-\log x$ is $\infty$. Hence $\lim _{t \rightarrow \infty} \sup V(x(t))=\lim _{t \rightarrow \infty} \sup (x(t))-1-$ $\log (x(t))<\infty$ a.s.

Then it is not difficult to deduce that

$$
0<\lim _{t \rightarrow \infty} \inf x(t)<\lim _{t \rightarrow \infty} \sup x(t)<\infty \quad \text { a.s. }
$$

In conclusion, given any initial value $x_{0}$, the solution of (3) $x(t)$ will vary in the interval of $\left[\lim _{t \rightarrow \infty} \inf x(t)\right.$, $\left.\lim _{t \rightarrow \infty} \sup x(t)\right]$.

From Theorem 4, the stakeholders of the ecological tourism system with visitor educational intervention can develop within an interval sustainably and steadily. Different kinds of stakeholders retained in a safety interval can immigrate from outside the system or emigrate from inside the system with regular rate. Hence, visitor education programmes are important elements of management efforts to ensure the protection of protected areas including Natural History Park and precious culture resource areas, and so forth.

\section{The Simulations of the Stochastic Dynamic Model of Ecological Tourism System}

Following the Euler-Maruyama method [22] and using Matlab program, we can obtain the numerical solutions and their figures of the stochastic dynamic model of ecological tourism system (5) with different parameters $\alpha, \beta, \gamma$ and different initial values $x_{0}$.

Given $\alpha=0.25, \beta=0.12, \gamma=0.08$, and $K=1000$, it is easy to gain the simulation data of $x(t)$ and draw Figure 3 when $x_{0}=30$ and $x_{0}=100$. Figure 3 demonstrates that the stochastic dynamic model is varying far away from the maximum carrying capacity of the ecological system 
$K=1000$. And the peak value of $x(t)$ is 625 much smaller than $K=1000$. Comparing Figure 1 with Figure 3, it can be concluded that the stochastic dynamic model (5) can effectively suppress the explosion and avoid the chaotic and unsustainable situation with the same parameters $\alpha, \beta, \gamma$.

Given $\alpha=0.15, \beta=0.08, \gamma=0.06, K=1000$, it is not difficult to get the simulation data of $x(t)$ and draw Figure 4 when $x_{0}=30$ and $x_{0}=80$. Figure 2 shows that the original logistical differential Equation (1) collapses in a finite time. But Figure 4 shows that the stochastic dynamic model (5) does not explode with the same parameters $\alpha, \beta, \gamma$. The peak value of $x(t)$ is 298 with smaller parameters $\alpha, \beta, \gamma$, see Figure 4.

Shown by Figure 5, the ecological tourism system perturbed by visitor educational intervention does not collapse even with larger initial value $x_{0}=900$ which is very close to $K=1000$. When there is a large number of visitors in the ecological tourism system, the effective visitor education programme will also keep resource and social conditions within acceptable limits by employing low impact behaviors. Figure 5 demonstrates that the system restores to normal condition quickly. Following Figures 3 to 5, it can be concluded that the ecological tourism system with visitor educational intervention can develop safely and steadily.

\section{Conclusions}

The ever-increasing demand for outdoor recreation in parks and protected areas worldwide continues to present a considerable challenge for managers [22]. Allowing recreational use while preserving natural conditions, it also presents a challenge for park managers in China because of fast expanding visitation. The protected areas including some natural history parks will be quickly damaged without visitor education intervention, while regulation and site management are also gaining very limited efficacy.

In Section 2, supposing that the different kinds of stakeholders of ecological tourism system grow with the specified intrinsic increasing rate and the stakeholders from outside competitors can immigrate into the system and some insider of the system can also go out freely when they finish their activities or business, we set up a differential equation to describe the ecological tourism system. Without any perturbed factors especially visitor educational intervention shown by the theoretical proof and numerical simulation, the solution of the dynamic system will explode in a finite time given any initial value.

In order to avoid the explosion of the solution of the dynamic model and the unstable situation of the ecological tourism system, we add visitor educational intervention into the system in Section 3. Supposing that the intrinsic increasing rate of stakeholders is stochastically perturbed by the visitor educational intervention, we explore a stochastic dynamic model which can effectively suppress the explosion of the solution shown in Section 4. And in Section 5, we also prove that the solution of the stochastic dynamic model will vary in a finite interval. In Section 6, following the EulerMaruyama method, we obtain the numerical solutions and their figures of the stochastic dynamic model of ecological

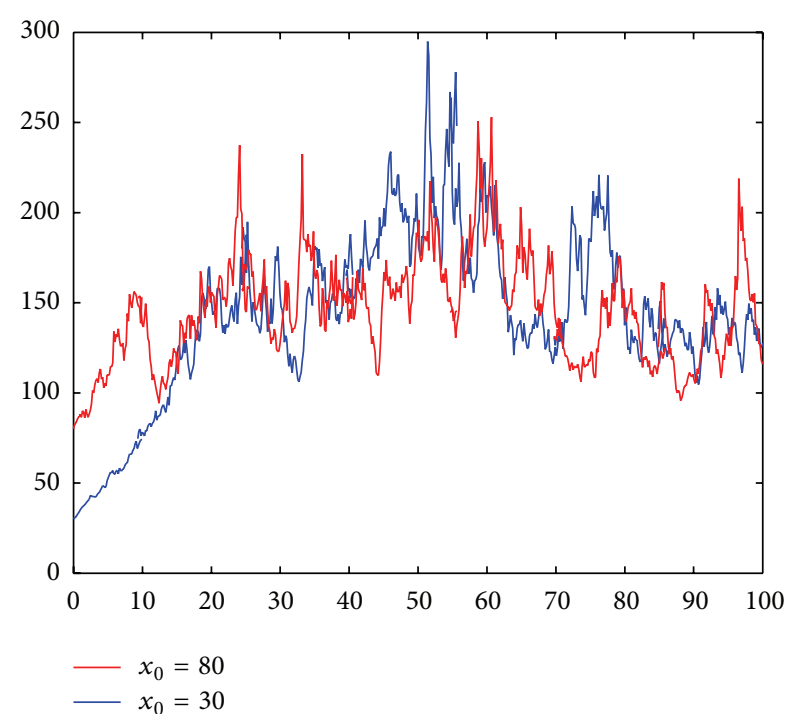

FIgURE 4: The simulation of $x(t)$ with $\alpha=0.15, \beta=0.08, \gamma=0.06$, $K=1000$ and different initial value $x_{0}$.

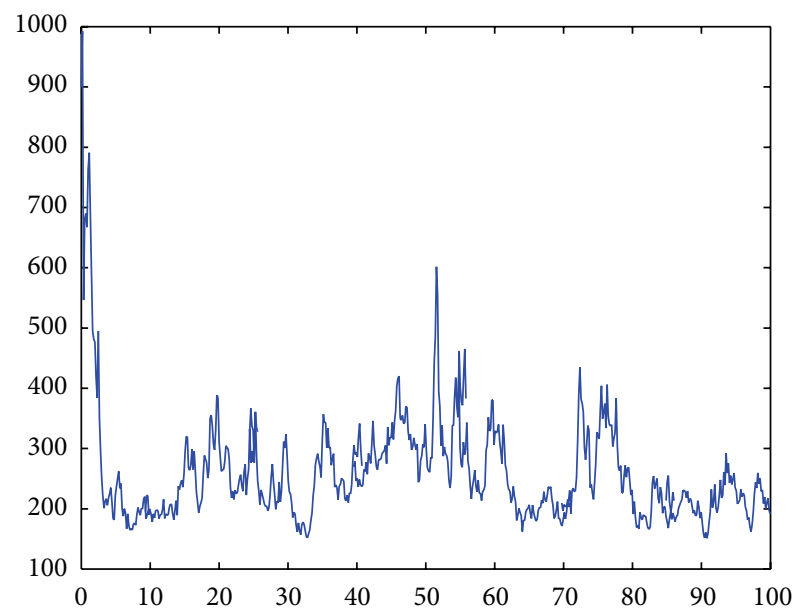

Figure 5: The simulation of $x(t)$ with $\alpha=0.25, \beta=0.12, \gamma=0.08$, $K=100$ and $x_{0}=900$.

tourism system. So we demonstrate that the tourism system will develop steadily and safely even under a large amount of visitors in public vocation, when employing continuous visitor education intervention programmes.

Generally, the findings of the stochastic dynamic model support that the continuous visitor education intervention including ecological knowledge, minimum impact behavior or technique, and regulations message is the necessary response to the challenge of recreation impact issues which try to manage resources sustainably and provide quality visitor experiences.

\section{Authors' Contribution}

Dongping Wei and Shouwen Wen equally contributed to this work. 


\section{Acknowledgments}

This work is supported by National Social Science Foundation of China (Grant no. 11BGL053), National Science Fund of China (Grant no. 71272177/G020902, Grant No. 11101434), Shenzhen Education Society Fund (Grant no. XH021). And the authors would like to thank the editor and anonymous referees for their helpful comments on the paper.

\section{References}

[1] China National Tourism Administration, The Yearbook of China Tourism Statistics, Travel \& Tourism Press, Beijing, China, 2012.

[2] W. E. Hammitt and D. N. Cole, Wildland Recreation: Ecology and Managemnet, John Wiley, New York, NY, USA, 2nd edition, 1998.

[3] J. C. Hendee and C. P. Dawson, "Wilderness visitor management: stewardship for quality experiences," in Wilderness Management: Stewardship and Protection of Resources and Values, Fulcrum, Golden, Colo, USA, 2002.

[4] R. E. Manning, Studies in Outdoor Recreation: Search and Research for Satisfaction, Oregon State University Press, Corvallis, Ore, USA, 1999.

[5] M. N. Ferreira and S. Rosso, "Effects of human trampling on a rocky shore fauna on the Sao Paulo coast, southeastern Brazil," Brazilian Journal of Biology, vol. 69, no. 4, pp. 993-999, 2009.

[6] R. E. Manning and L. E. Andrson, "how many visitors is too many at Arches?" in Managing Outdoor Recreation: Case Studies in the National Parks, pp. 71-75, 2012.

[7] J. L. Marion and T. A. Farrell, "Management practices that concentrate visitor activities: camping impact management at Isle Royale National Park, USA," Journal of Environmental Management, vol. 66, no. 2, pp. 201-212, 2002.

[8] G. L. Peterson and D. W. Lime, "People and their behavior: a challenge for recreation management," Journal of Forestry, vol. 77, no. 6, pp. 343-347, 1979.

[9] G. H. Stankey and R. Schreyer, "Attitudes towards wilderness and factors affecting visitor behavior:A state of knowledge review," in Proceedings of the National Wilderness Research Conference. Issues, State of Knowledge, Future. Directions, R. C. Lucas, Ed., General Technical Report INT-220, pp. 246-293, USDA Forest Service, Intermountain Research Station, Ogden, Utah, USA, 1987.

[10] J. L. Marion and S. E. Reid, "Minimising visitor impacts to protected areas: The efficacy of low impact education programmes," Journal of Sustainable Tourism, vol. 15, no. 1, pp. 5-27, 2007.

[11] J. E. Doucette and D. N. Cole, "The wilderness visitor education: Information about alternative techniques," General Technical Report-Intermountain Research Station, USDA Forest Service No.INT-2995, 1993.

[12] W. Shou-wen, "On the growth of stakeholders in ecotourism destination," Journal of Wuhu Vocational Institute of Technology, vol. 11, no. 1, pp. 1-3, 2009 (Chinese).

[13] W. Shou-wen, X. Yong, and W. Dongping, "Stochastic ecological tourism system dynamic model with visitor educational intervention," Wroking Paper, 2012.

[14] X. Mao, G. Marion, and E. Renshaw, "Environmental Brownian noise suppresses explosions in population dynamics," Stochastic Processes and their Applications, vol. 97, no. 1, pp. 95-110, 2002.

[15] G. S. Ladde and V. Lakshmikantham, Random Differential Inequalities, vol. 150, Academic Press, New York, NY, USA, 1980.
[16] R. Liptser and A. N. Shiryayev, Theory of Martingales, Kluwer Academic, Dordrecht, The Netherlands, 1989, Translation of the Russian Edition, Nauka, Moscow.

[17] X. Mao, Stochastic Differential Equations and Applications, Horwood, New York, NY, USA, 1997.

[18] X. Mao, Stochastic Differential Equations and Applications, Harwood, Chichester, UK, 2007.

[19] A. Friedman, Stochastic Differential Equations and their Applications, Academic Press, New York, NY, USA, 1976.

[20] J. R. Fazio, Communicating with the Wilderness User. Bulletin Number 28, University of Idaho, College of Forestry, Wildlife and Range Experiment Station, Moscow, Idaho, USA, 1979.

[21] J. A. Bradley, "A human approach to reducing wildland impacts. Proceedings-Recreational Impacts on Wildlands (P. 222-6)," Tech. Rep. R-6-001-1970, USDA Forest Service, Pacific Northwest Region, Portland, Ore, USA, 1979.

[22] P. E. Kloeden and E. Platen, Numerical Solution of Stochastic Differential Equations, Springer, Berlin, Germany, 1999. 


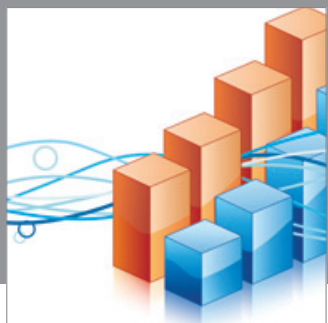

Advances in

Operations Research

mansans

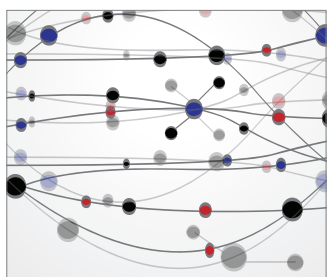

The Scientific World Journal
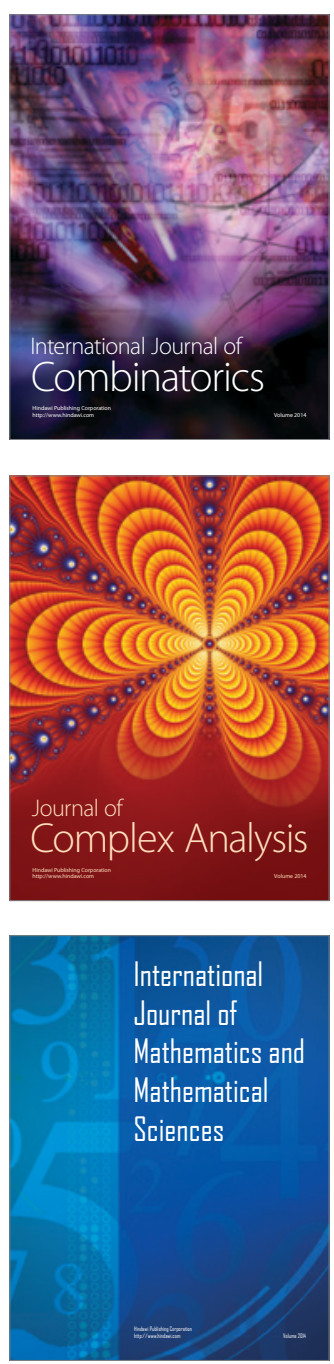
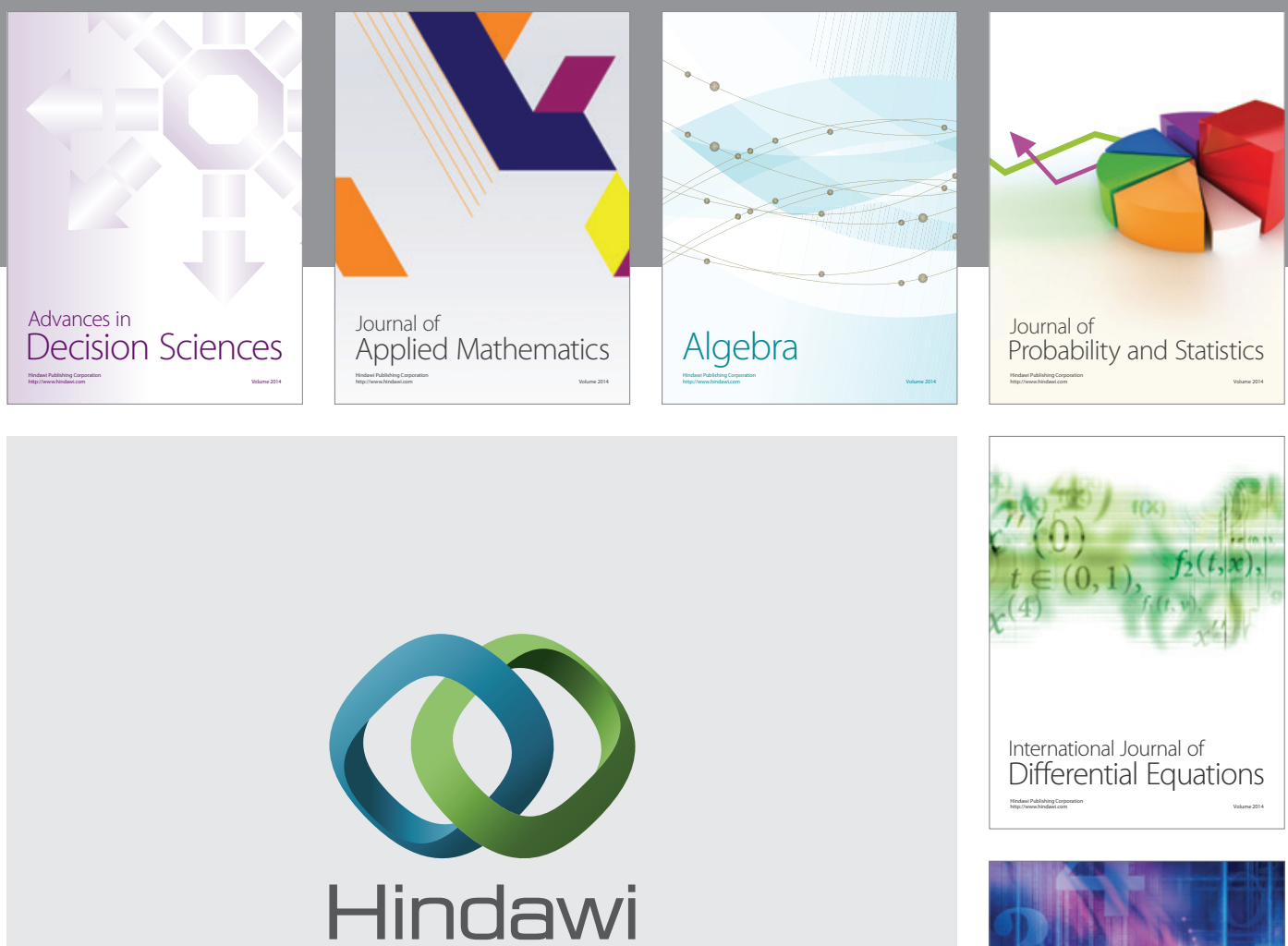

Submit your manuscripts at http://www.hindawi.com
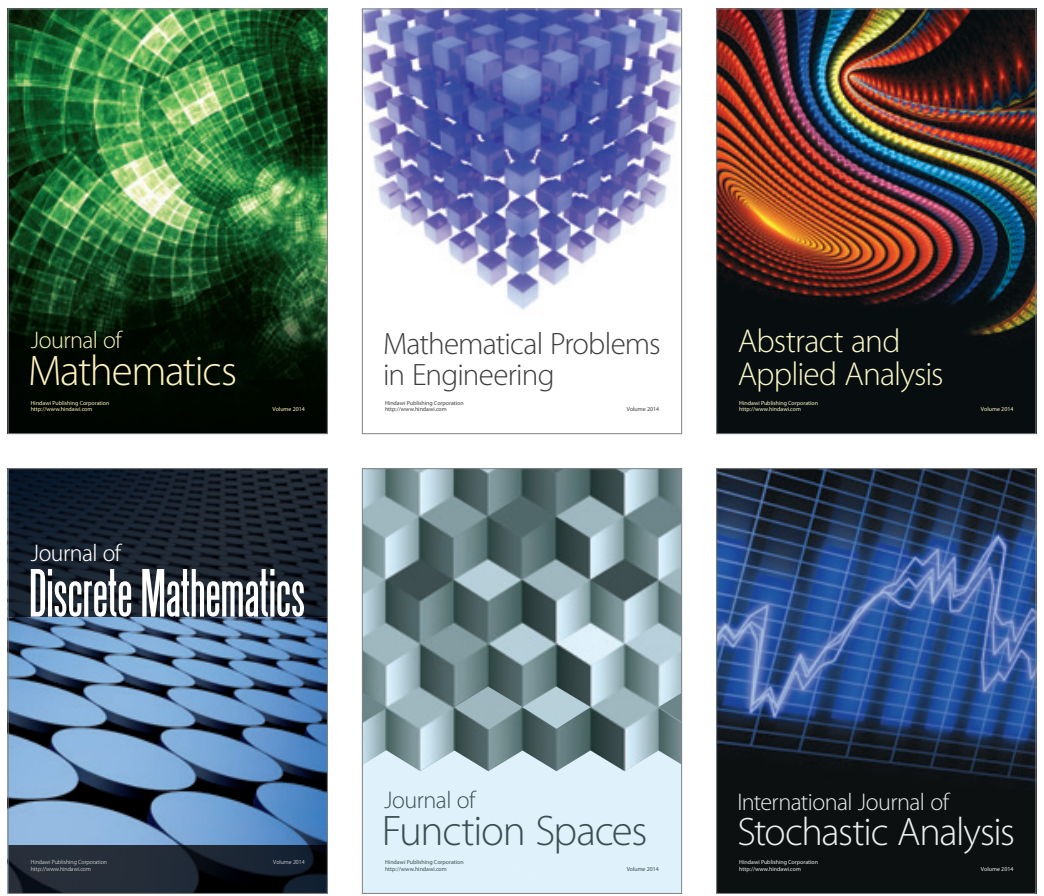

Journal of

Function Spaces

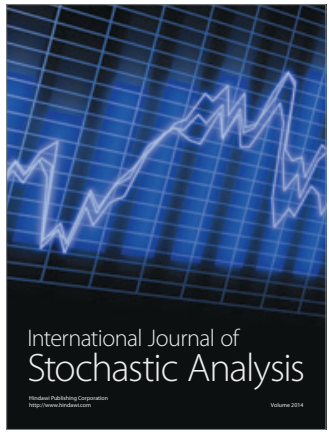

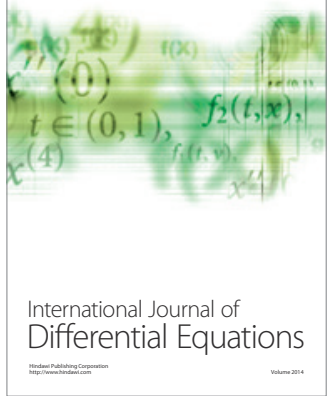
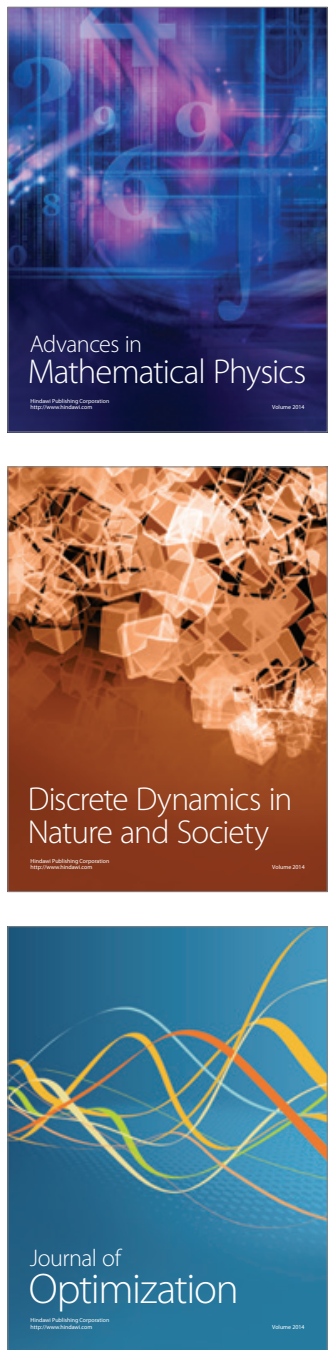